УДК 535.015

\title{
ВЫРОЖДЕННОЕ ПОЛЯРИЗАЦИОННОЕ МНОГОВОЛНОВОЕ ВЗАИМОДЕЙСТВИЕ СВЕТОВЫХ ПУЧКОВ В РАСТВОРЕ КРАСИТЕЛЯ РОДАМИН 6Ж
}

\author{
(C) 2013 г. Д. В. Горбач, С. А. Назаров, А. Л. Толстик \\ Белорусский государственный университет, Минск \\ E-mail: sanazarov@tut.by
}

\begin{abstract}
Исследованы характеристики дифрагированных волн, получаемых при четырех- и шестиволновом смешении в растворе красителя родамин 6Ж при различных комбинациях состояний поляризации, участвующих во взаимодействии волн.
\end{abstract}

DOI: $10.7868 / \mathrm{S} 0367676513120090$

\section{ВВЕДЕНИЕ}

Одной из актуальных задач современной оптической индустрии является поиск новых эффективных методов управления энергетическими, спектральными и поляризационными характеристиками лазерного излучения [1-4]. Наряду с использованием анизотропных нелинейных интерферометров [5, 6] большими потенциальными возможностями обладают голографические методы преобразования световых полей на основе динамических голограмм в растворах сложных органических соединений при многоволновых взаимодействиях (МВB) [7].

Цель работы - исследование зависимости эффективности четырех- и шестиволнового взаимодействий (ЧВВ и ШВВ соответственно) с гауссовой и сингулярной сигнальной волной от поляризации записывающих голограмму волн и установление поляризационных закономерно- стей для дифрагированной волны при различных комбинациях поляризаций, участвующих в многоволновом взаимодействии волн.

\section{ЭКСПЕРИМЕНТАЛЬНАЯ УСТАНОВКА}

Экспериментальная схема представлена на рис. 1. В качестве источника излучения использована вторая гармоника Nd:YAG-лазера 1. Лазер работал в режиме активной модуляции добротности с длительностью импульса 20 нс и частотой повторения 10 Гц. С помощью светоделителя 2 зеркала 3 , 4, 5 формировали соответственно считывающую, опорную и сигнальную волны, которые сводились в кювете с нелинейной средой (раствором красителя родамин 6Ж) 10, где и происходило МВВ. Линия задержки 6 предназначена для выравнивания оптических путей сигнальной и опорной волн. Для создания сингулярного сигнального пучка в схему вносили голографический транспарант 7.

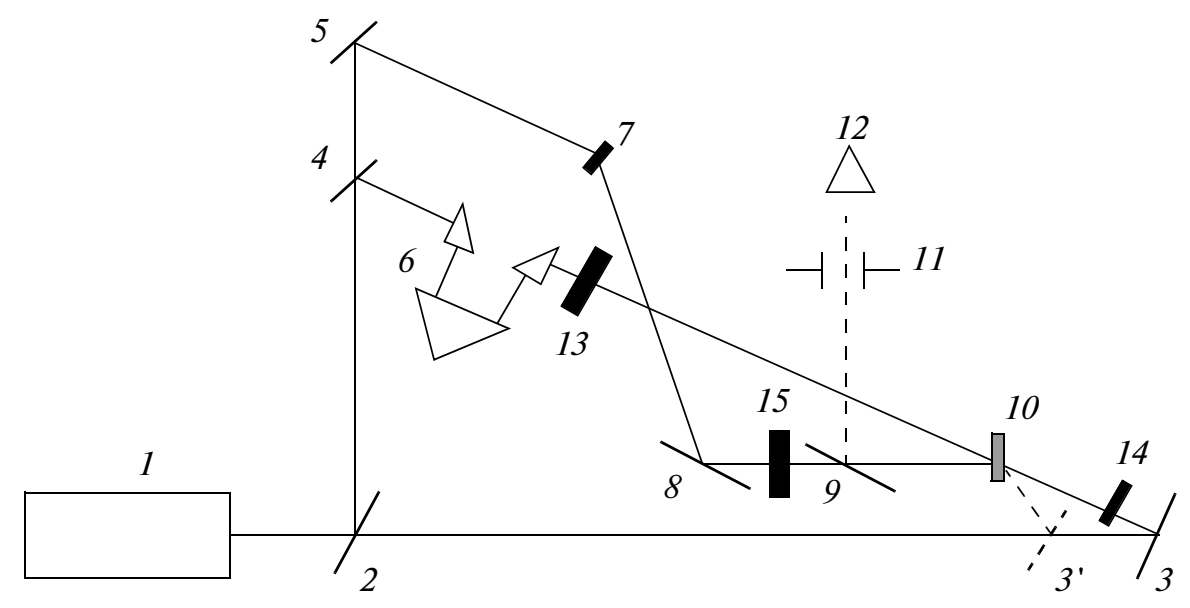

Рис. 1. Схема экспериментальной установки. 

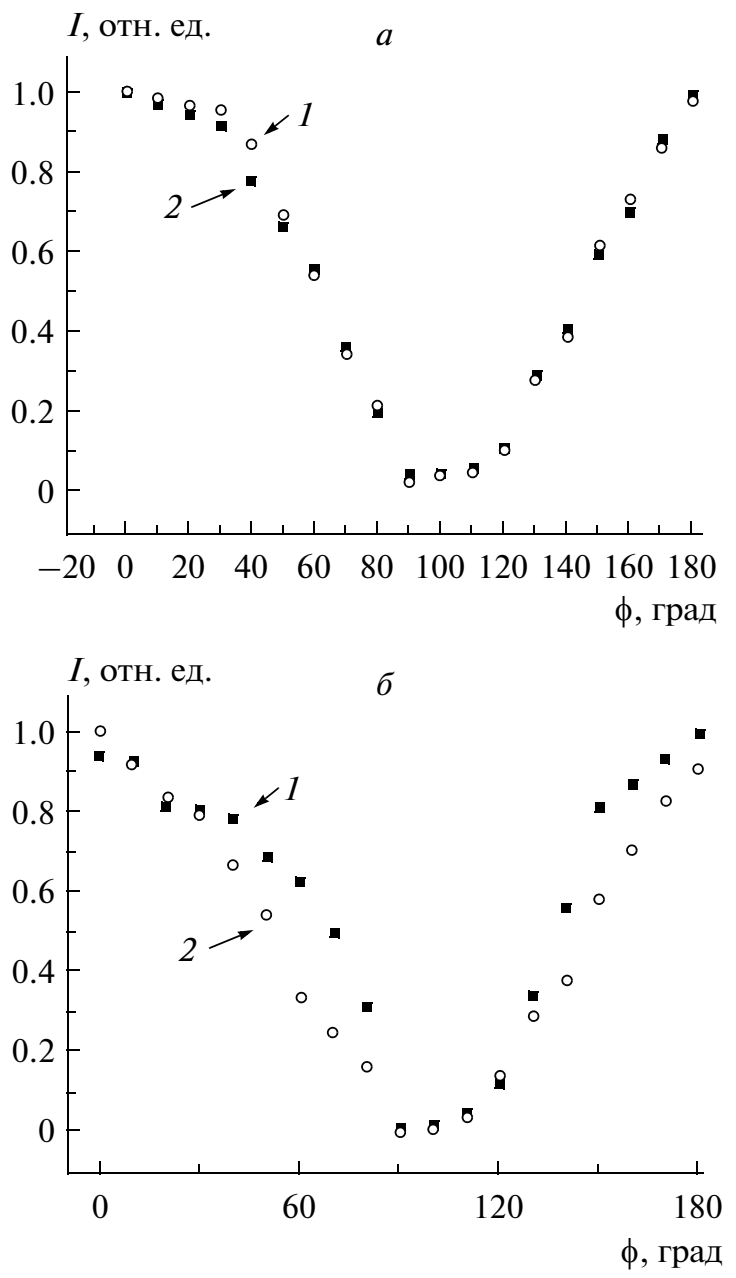

Рис. 2. Зависимости нормированной интенсивности $(I)$ дифрагированной волны от угла ( $\phi)$ между плоскостями поляризации участвующих в МВВ волн; $a: 1-$ при ЧВВ, 2 - при ШВВ; $\sigma: 1$ - ЧВВ с гауссовой сигнальной волной, 2 - ЧВВ с сингулярной сигнальной волной.

Зеркало 8 направляло полученный оптический вихрь в кювету с нелинейной средой. При распространении считывающей волны точно навстречу опорной реализуется ЧВВ. Для реализации ШВВ считывающая волна отклонялась перемещением зеркала 3 в положение 3' для достижения соответствующего условия фазового синхронизма. Зеркало 9 направляло дифрагированный пучок через диафрагму 11 в систему регистрации 12 . Для из-

Таблица 1. Экспериментальная зависимость поляризации дифрагированной волны при ЧВВ от состояний поляризации взаимодействующих волн

\begin{tabular}{|l|c|c|c|c|c|c|c|c|}
\hline \multicolumn{1}{c|}{ Волна } & \multicolumn{7}{c}{ Поляризация } \\
\hline Опорная & $\rightarrow$ & $\uparrow$ & $\rightarrow$ & $\rightarrow$ & $\uparrow$ & $\rightarrow$ & $\uparrow$ & $\uparrow$ \\
\hline Сигнальная & $\rightarrow$ & $\rightarrow$ & $\uparrow$ & $\rightarrow$ & $\uparrow$ & $\uparrow$ & $\rightarrow$ & $\uparrow$ \\
\hline Считывающая & $\rightarrow$ & $\rightarrow$ & $\rightarrow$ & $\uparrow$ & $\rightarrow$ & $\uparrow$ & $\uparrow$ & $\uparrow$ \\
\hline Дифрагированная & $\rightarrow$ & $\uparrow$ & $\uparrow$ & $\uparrow$ & $\rightarrow$ & $\rightarrow$ & $\rightarrow$ & $\uparrow$ \\
\hline
\end{tabular}

менения состояния поляризации волн в схему вносили пластинки $\lambda / 2$ и $\lambda / 4$ 13-15.

\section{ЭКСПЕРИМЕНТАЛЬНЫЕ РЕЗУЛЬТАТЫ}

Результаты исследований зависимости эффективности МВВ с гауссовой и сингулярной сигнальной волной от угла между плоскостями поляризации взаимодействующих волн представлены на рис. 2. Видно, что зависимости интенсивности дифрагированной волны от угла между плоскостями поляризации сигнальной и опорной волн для ЧВВ и ШВВ с гауссовой сигнальной волной практически совпадают (рис. $2 a$ ). Чем ближе угол между плоскостями поляризации сигнальной и опорной волн к 90 градусам, тем меньше эффективность МВВ. При ортогональной поляризации сигнальной и опорной волн эффективность МВВ минимальна. Это объясняется тем, что в данном случае отсутствует пространственная модуляция интенсивности и имеет место только модуляция поляризации света в соответствии с разностью фаз между волнами. Свет с разными поляризациями (например, линейной и круговой) по-разному возбуждает молекулы красителя и индуцирует периодическую модуляцию комплексного показателя преломления. При этом реализуются условия поляризационной голографической записи [8]. Как показали экспериментальные результаты, эффективность МВВ на поляризационных голографических решетках примерно в 50 раз ниже, чем при МВВ с одинаковой ориентацией плоскостей поляризации взаимодействующих волн (рис. 2a).

При МВВ с использованием сингулярной сигнальной волны зависимость в целом аналогична. Однако следует отметить, что более сложная реализация эффективного перекрытия гауссова и сингулярного пучков проявляется в более высокой чувствительности МВВ к изменению угла между плоскостями поляризации сингулярной сигнальной и гауссовой опорной волн по сравнению со случаем взаимодействия всех гауссовых световых пучков (рис. 2б).

Изменяя ориентации поляризаций всех взаимодействующих волн определили состояния поляризации дифрагированной волны при МВВ (табл. 1 и 2). Видно, что при реализации взаимо-

Таблица 2. Экспериментальная зависимость поляризации дифрагированной волны при ШВВ от состояний поляризации взаимодействующих волн

\begin{tabular}{|l|c|c|c|c|c|c|c|c|}
\hline \multicolumn{1}{c|}{ Волна } & \multicolumn{7}{c}{ Поляризация } \\
\hline Опорная & $\rightarrow$ & $\uparrow$ & $\rightarrow$ & $\rightarrow$ & $\uparrow$ & $\rightarrow$ & $\uparrow$ & $\uparrow$ \\
\hline Сигнальная & $\rightarrow$ & $\rightarrow$ & $\uparrow$ & $\rightarrow$ & $\uparrow$ & $\uparrow$ & $\rightarrow$ & $\uparrow$ \\
\hline Считывающая & $\rightarrow$ & $\rightarrow$ & $\rightarrow$ & $\uparrow$ & $\rightarrow$ & $\uparrow$ & $\uparrow$ & $\uparrow$ \\
\hline Дифрагированная & $\rightarrow$ & $\rightarrow$ & $\rightarrow$ & $\uparrow$ & $\rightarrow$ & $\uparrow$ & $\uparrow$ & $\uparrow$ \\
\hline
\end{tabular}


действия на решетках, записанных одинаково поляризованными волнами, поляризация дифрагированной волны как при ЧВВ, так и при ШВВ определяется поляризацией считывающей волны. Иная ситуация имеет место при реализации MBВ на поляризационных динамических решетках. В этом случае поляризация дифрагированной волны зависит не только от поляризации считывающей волны, но и от порядка взаимодействия. При ЧВВ имеет место поворот плоскости поляризации считывающей волны на 90, а при ШВВ поляризация дифрагированной волны совпадает с поляризацией считывающей волны. Указанная закономерность определяется особенностями дифракции на поляризационных решетках в первый (ЧВВ) и второй (ШВВ) порядки и совпадает с данными, приведенными в [9] при рассмотрении взаимодействия гауссовых сигнальной, опорной и считывающей волн.

Использование нами сингулярного пучка, имеющего винтовую фазовую дислокацию, не привело к каким-либо изменениям и подтвердило независимость особенностей преобразования поляризации при дифракции на поляризационных динамических решетках от вида сигнального пучка (гауссова и сингулярного), несмотря на наличие орбитального момента.

Также представляет интерес рассмотрение МВВ волн с круговой поляризацией. На рис. $3 a$ представлены экспериментальные результаты, полученные для ЧВВ при круговой поляризации одной из взаимодействующих волн и вертикальных линейных поляризаций остальных. Показано, что при круговой поляризации опорной или сигнальной волн поляризация дифрагированной волны является эллиптической, сильно вытянутой вдоль вертикального направления. Такая ситуация объяснена следующим образом. Круговая поляризация может быть представлена как сумма двух ортогональных линейно поляризованных волн со сдвигом фаз между ними. Вертикальные составляющие поляризации записывающих голограмму волн образуют классическую динамическую решетку, при дифракции на которой реализуется традиционное многоволновое взаимодействие с образованием дифрагированной волны с вертикальной поляризацией. Горизонтальная составляющая круговой поляризации участвует в записи поляризационной решетки. На ней также происходит многоволновое взаимодействие, которое дает вклад в горизонтальную составляющую поляризации дифрагированной волны. Однако, как отмечено выше, эффективность такого взаимодействия на один - два порядка меньше, поэтому и горизонтальная составляющая поляризации дифрагированной волны намного меньше вертикальной.

При взаимодействии опорной и сигнальной волн с ортогональными круговыми поляризация-

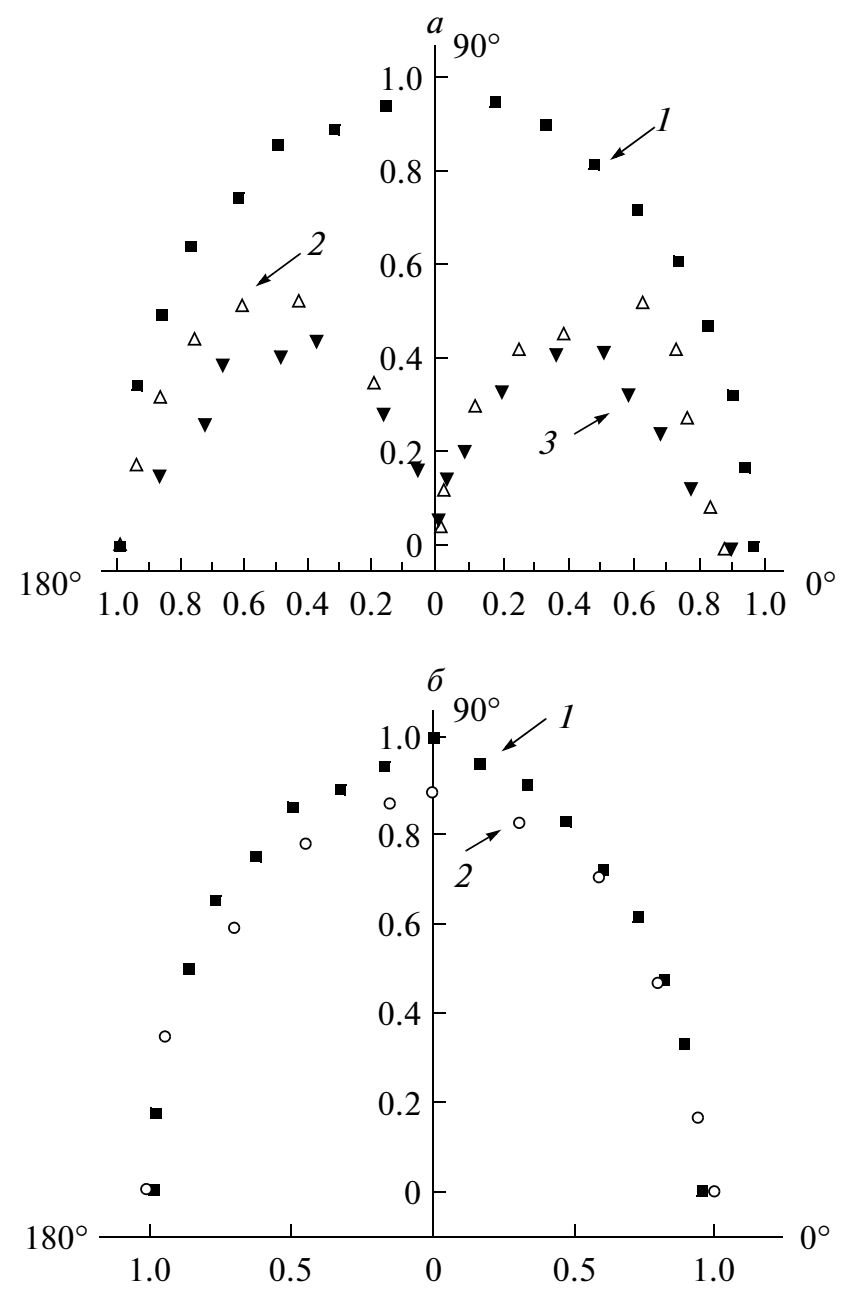

Рис. 3. Индикатриса состояния поляризации дифрагированной волны при ЧВВ; $a: 1-$ круговое состояние поляризации, 2 - состояние поляризации дифрагированной волны при круговой поляризации сигнальной волны, 3 - состояние поляризации дифрагированной волны при круговой поляризации опорной волны; б: 1 - круговое состояние поляризации, 2 - состояние поляризации дифрагированной волны при ортогональных круговых поляризациях сигнальной и опорной волн.

ми происходит запись поляризационной динамической решётки, что проявляется в формировании дифрагированной волны с круговой поляризацией при считывании решетки волной с линейной поляризацией (рис. 3б).

\section{ЗАКЛЮЧЕНИЕ}

Полученные результаты позволили исследовать зависимость эффективности многоволновых взаимодействий как с гауссовой, так и с сингулярной сигнальной волной от угла между плоскостями поляризаций записывающих динамическую голограмму волн. Получены зависимости состояния поляризации дифрагированной волны при много- 
волновых взаимодействиях волн как с линейной, так и с круговой поляризацией. Определены состояния поляризации дифрагированной волны при различных комбинациях поляризаций всех взаимодействующих волн. Экспериментально подтверждена зависимость поляризации от вида взаимодействия (ЧВВ и ШВВ) при использовании сингулярной волны в качестве сигнального пучка.

\section{СПИСОК ЛИТЕРАТУРЫ}

1. Varhegyi P., Kerekes A., Sajti S. et al. // Appl. Phys. 2003. V. 76. P. 397.

2. Hiroshi O., Akira E., Nobuhiro K., Takako H. // Opt. Exp. 2003. V. 11. № 19. P. 2379.

3. Sarik R.N., Nelson V.T., Diane M.S. Brian R.K. // Appl. Opt. 2009. V. 48. № 21. P. 4062.
4. Deyin Z., Hongjun Y., Zhenqiang M., Weidong Z. // Opt. Exp. 2011. V. 19. № 10. P. 9050.

5. Gancherenok I.I., Romanov O.G., Tolstik A.L. et al. // J. of Optics B: Quantum and Semiclassical Optics. 2001. V. 3. P. S220.

6. Romanov O.G., Tolstik A.L., Ormachea O. et al. // Laser Optics 2003: Wavefront Transformation and Laser Beam Control: Proc. SPIE. 2004. V. 5481. P. 90.

7. Rubanov A.S., Tolstik A.L., Karpuk S.M., Ormachea O. // Opt. Commun. 2000. V. 181. P. 183.

8. Blanche P.-A., Lemaire Ph.C., Maertens C. et al. // Opt. Commun. 2000. V. 185. P. 1.

9. Ормачеа О., Толстик А.Л. // Изв. РАН. Сер. физ. 2005. T. 69. № 8. C. 1144; Ormachea O., Tolstik A.L. // Bull. Russ. Acad. Sci.: Physics. 2005. V. 69. № 8. P. 1280 . 